\title{
AIRY FUNCTIONS AND APPLICATIONS TO PHYSICS
}


This page intentionally left blank 


\section{AIRY FUNCTIONS AND APPLICATIONS TO PHYSICS}

\section{Olivier Vallée \\ Université d'Orléans, France \\ Manuel Soares}

Ministère de l'Équipement, D D E des Yvelines, France 
Published by

Imperial College Press

57 Shelton Street

Covent Garden

London WC2H 9HE

Distributed by

World Scientific Publishing Co. Pte. Ltd.

5 Toh Tuck Link, Singapore 596224

USA office: 27 Warren Street, Suite 401-402, Hackensack, NJ 07601

UK office: 57 Shelton Street, Covent Garden, London WC2H 9HE

\section{British Library Cataloguing-in-Publication Data}

A catalogue record for this book is available from the British Library.

\section{AIRY FUNCTIONS AND APPLICATIONS TO PHYSICS}

Copyright $\mathcal{O} 2004$ by Imperial College Press

All rights reserved. This book, or parts thereof, may not be reproduced in any form or by any means, electronic or mechanical, including photocopying, recording or any information storage and retrieval system now known or to be invented, without written permission from the Publisher.

For photocopying of material in this volume, please pay a copying fee through the Copyright Clearance Center, Inc., 222 Rosewood Drive, Danvers, MA 01923, USA. In this case permission to photocopy is not required from the publisher.

ISBN $\quad 1-86094-478-7$ 


\section{Preface}

The use of special functions, and in particular of Airy functions, is rather common in physics. The reason may be found in the need, and even in the necessity, to express a physical phenomenon in terms of an effective and comprehensive analytical form for the whole scientific community. However, for almost the last twenty years, many physical problems have been resolved by computers. This trend is now becoming the norm as the importance of computers continues to grow. As a last resort, the special functions employed in physics will have, indeed, to be calculated numerically, even if the analytic formulation of physics is of first importance.

The knowledge on Airy functions was periodically the subject of many review articles. Generally these were about their tabulations for the numerical calculation of these functions which is particularly difficult. We shall quote the most known works in this field: the tables of J.C.P. Miller which are from 1946 and the chapter in the Handbook of Mathematical Functions by Abramowitz and Stegun whose first version appeared in 1954. No noteworthy compilation on Airy functions has been published since that time, in particular about the calculus implying these functions. For example, in the last editions of the tables of Gradshteyn and Ryzhik, they are hardly evoked. At the same time, many accumulated results in the scientific literature, remain extremely dispersed and fragmentary.

The Airy functions are used in many fields of physics, but the analytical outcomes that have been obtained are not (or weakly) transmitted between the various fields of research which after all remain isolated. Moreover the tables of Abramowitz and Stegun are still the only common reference to all the authors using these functions. Thus many of the results have been rediscovered, sometimes extremely old findings are the subject of publications and consequently a useless effort for researchers. 
In this work, we would like to make a rather exhaustive compilation of the current knowledge on the analytical properties of Airy functions. In particular, the calculus implying the Airy functions is developed with care. This is, actually, one of the major objectives of this book. We are however aware of making a great number of repetitions regarding the previous compilations, but, it seemed necessary to ensure coherence. This book is addressed mainly to physicists (from undergraduate students to researchers). For the mathematical demonstrations, as one will see, we do not have any claim about the rigour. ${ }^{1}$ The aim is the outcome, or the fastest way to reach it. Finally, in the second part of this work, the reader will find some applications to various fields of physics. These examples are not exhaustive. They are only given to succinctly illustrate the use of Airy functions in classical or in quantum physics. For instance, we point out to the physicist in fluid mechanics, that he can find what he is looking for, in the works of molecular physics or in physics of surfaces, and vice versa.

The authors would like to warmly thank Nick Rowswell who considerably improved the content of this book.

O. Vallée \& M. Soares, Fall 2003

\footnotetext{
${ }^{1}$ As a matter of fact, the Airy function can be considered as a distribution (generalised function) whose Fourier transform is an imaginary exponential. Also most of the integrals evoked in this work should be evaluated with the help of a convergence factor.
} 


\section{Contents}

Preface $\quad$ v

1. A Historical Introduction : Sir George Biddell Airy 1

2. Definitions and Properties 5

2.1 The Homogeneous Airy Functions . . . . . . . . . 5

2.1.1 The Airy's equation ............ . . 5

2.1.2 Elementary properties . . . . . . . . . 8

2.1.2.1 Wronskians of homogeneous Airy functions. 8

2.1.2.2 Particular values of Airy functions . . . . 8

2.1.2.3 Relations between Airy functions . . . . . . 9

2.1.3 Integral representations . . . . . . . . . . 9

2.1.4 Ascending and asymptotic series . . . . . . . 11

2.1.4.1 Expansion of $A i$ near the origin . . . . . . 11

2.1.4.2 Ascending series of $A i$ and $B i \ldots \ldots 12$

2.1.4.3 Asymptotic series of $A i$ and $B i \ldots \ldots$

2.2 Properties of Airy Functions . . . . . . . . . . 15

2.2 .1 Zeros of Airy functions . . . . . . . . . 15

2.2 .2 The spectral zeta function . . . . . . . . 18

2.2 .3 Inequalities . . . . . . . . . . . . . 20

2.2.4 Connection with Bessel functions . . . . . . . . 20

2.2.5 Modulus and phase of Airy functions . . . . . . . 21

2.2.5.1 Definitions . . . . . . . . . . . 21

2.2.5.2 Differential equations . . . . . . . . . . . . . 22

2.2.5.3 Asymptotic expansions . . . . . . . . . . . 23

2.2.5.4 Functions of positive arguments . . . . . . 24 
2.3 The Inhomogeneous Airy Functions . . . . . . . . . . . 25

2.3 .1 Definitions . . . . . . . . . . . . . 25

2.3.2 Properties of inhomogeneous Airy functions . . . . 27

2.3.2.1 Values at the origin . . . . . . . . 27

2.3.2.2 Other integral representations . . . . . . 27

2.3.3 Ascending and asymptotic series . . . . . . . . 28

2.3.3.1 Ascending series . . . . . . . . . . . . . 28

2.3.3.2 Asymptotic series . . . . . . . . . . . . . . . . 29

2.3.4 Zeros of the Scorer functions . . . . . . . . . . . . 29

2.4 Squares and Products of Airy Functions . . . . . . . . . 30

2.4.1 Differential equation and integral representation . . 30

2.4 .2 A remarkable identity . . . . . . . . . . . . 32

2.4.3 The product $A i(x) A i(-x)$ : Airy wavelets . . . . 32

3. Primitives and Integrals of Airy Functions $\quad 37$

3.1 Primitives Containing One Airy Function $\ldots \ldots \ldots$

3.1.1 In terms of Airy functions . . . . . . . . 37

3.1 .2 Ascending series . . . . . . . . . . . . 38

3.1 .3 Asymptotic series . . . . . . . . . . . . . . 38

3.1 .4 Primitive of Scorer functions . . . . . . . . . . . 39

3.1 .5 Repeated primitives . . . . . . . . . . 40

3.2 Product of Airy Functions . . . . . . . . . . . . . 40

3.2 .1 The method of Albright . . . . . . . . . . . 41

3.2 .2 Some primitives . . . . . . . . . . . . 43

3.3 Other Primitives $\ldots \ldots \ldots \ldots \ldots$. . . . . . . . . . 48

3.4 Miscellaneous . . . . . . . . . . . . . . . . . . . . 49

3.5 Elementary Integrals . . . . . . . . . . . . . 50

3.5.1 Particular integrals . . . . . . . . . . . 50

3.5.2 Integrals containing a single Airy function . . . . . 51

3.5.2.1 Integrals involving algebraic functions . . . . 51

3.5.2.2 Integrals involving transcendental functions 54

3.5.3 Integrals of products of two Airy functions . . . . . 56

3.6 Other Integrals . . . . . . . . . . . . . . . 60

3.6.1 Integrals involving the Volterra $\mu$-function . . . . . 60

3.6.2 Canonisation of cubic form . . . . . . . . . . 64

3.6.3 Integrals with three Airy functions . . . . . . 65

3.6.4 Integrals with four Airy functions . . . . . . . . . 67

3.6 .5 Double integrals . . . . . . . . . . . . . . 68 
4. Transformations of Airy Functions 71

4.1 Causal Properties of Airy Functions . . . . . . . . . 71

4.1 .1 Causal relations . . . . . . . . . . . . 71

4.1.2 Green function of the Airy equation . . . . . . 73

4.2 The Airy Transform . . . . . . . . . . . . . . . 74

4.2.1 Definitions and elementary properties . . . . . . . 74

4.2 .2 Some examples . . . . . . . . . . . . . . 77

4.2 .3 Airy polynomials . . . . . . . . . . . . 82

4.2.4 Summary of Airy transform . . . . . . . . . . 84

4.2 .5 Airy averaging . . . . . . . . . . . 85

4.3 Other Kinds of Transformations . . . . . . . . . . . 85

4.3.1 Laplace transform of Airy functions . . . . . . . 85

4.3.2 Mellin transform of Airy function . . . . . . . . 86

4.3.3 Fourier transform of Airy functions . . . . . . . 87

4.4 Expansion into Fourier-Airy Series . . . . . . . . . 88

5. The Uniform Approximation 91

5.1 Oscillating Integrals . . . . . . . . . . . . . . . 91

5.1.1 The method of stationary phase . . . . . . . 91

5.1.2 The uniform approximation of oscillating integral . . 93

5.1.3 The Airy uniform approximation . . . . . . . . . 94

5.2 Differential Equation of the Second Order . . . . . . 95

5.2 .1 The JWKB method . . . . . . . . . . . 95

5.2 .2 The generalisation of Langer . . . . . . . . . . . . . 97

5.3 Inhomogeneous Differential Equations . . . . . . . . 98

6. Generalisation of Airy Functions 101

6.1 Generalisation of the Airy Integral . . . . . . . . . . 101

6.2 Third Order Differential Equations . . . . . . . . . 105

6.2.1 The linear third order differential equation . . . . . 105

6.2 .2 Asymptotic solutions . . . . . . . . . . . 106

6.2.3 The comparison equation . . . . . . . . 107

6.3 Differential Equation of the Fourth Order . . . . . . . . . 111

7. Applications to Classical Physics 115

7.1 Optics and Electromagnetism . . . . . . . . . . 115

7.2 Fluid Mechanics . . . . . . . . . . . . . . . 119

7.2 .1 The Tricomi equation . . . . . . . . . . . 119 
7.2.2 The Orr-Sommerfeld equation . . . . . . . . 121

7.3 Elasticity . . . . . . . . . . . . . . . . . . . 124

7.4 The Heat Equation . . . . . . . . . . . . . . . 127

7.5 Nonlinear Physics . . . . . . . . . . . . . 129

7.5 .1 Korteweg-de Vries equation . . . . . . . . . . 129

7.5.1.1 The linearised Korteweg-de Vries equation . 129

7.5.1.2 Similarity solutions . . . . . . . . . 131

7.5 .2 The second Painlevé equation . . . . . . . . . . . . 132

7.5.2.1 The Painlevé equations . . . . . . . . . 132

7.5.2.2 An integral equation . . . . . . . . . . 134

7.5.2.3 Rational solutions . . . . . . . . . . 135

8. Applications to Quantum Physics 137

8.1 The Schrödinger Equation . . . . . . . . . . . . 137

8.1 .1 Particle in a uniform field . . . . . . . . 137

8.1 .2 The $|x|$ potential . . . . . . . . . . . . . 140

8.1.3 Uniform approximation of the Schrödinger equation . 144

8.1.3.1 The JWKB approximation . . . . . . . . 145

8.1.3.2 The Airy uniform approximation . . . . . 146

8.1.3.3 Exact vs approximate wave functions . . . 148

8.2 Evaluation of the Franck-Condon Factors . . . . . . . 152

8.2.1 The Franck-Condon principle . . . . . . . . . 153

8.2.2 The JWKB approximation . . . . . . . . . . . 154

8.2.3 The uniform approximation . . . . . . . . 157

8.3 The Semiclassical Wigner Distribution . . . . . . . . . 162

8.3.1 The Weyl-Wigner formalism . . . . . . . . . . 163

8.3.2 The one-dimensional Wigner distribution . . . . . . 164

8.3.3 The two-dimensional Wigner distribution . . . . 166

8.3.4 Configuration of the Wigner distribution . . . . . . 169

8.4 Airy Transform of the Schrödinger Equation . . . . . . . 173

Appendix A Numerical Computation of the Airy Functions 177

A.1 The Homogeneous Functions . . . . . . . . . . . . 177

A.2 The Inhomogeneous Functions . . . . . . . . . . 180

$\begin{array}{ll}\text { Bibliography } & 183\end{array}$

Index 